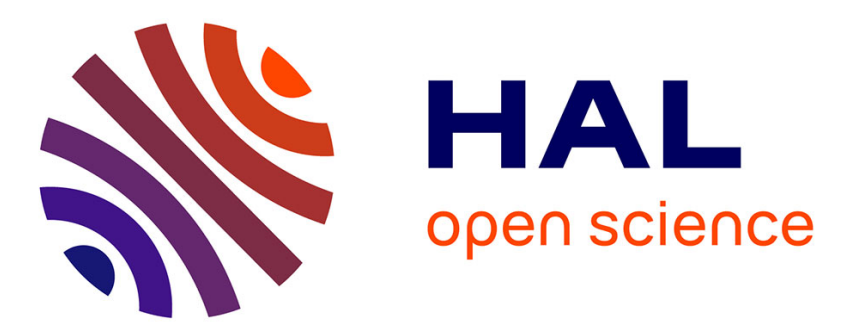

\title{
Altruism, Cheating, and Anticheater Adaptations in Cellular Slime Molds
}

Richard Ellis Hudson, Juliann Eve Aukema, Claude Rispe, Denis Roze

\section{To cite this version:}

Richard Ellis Hudson, Juliann Eve Aukema, Claude Rispe, Denis Roze. Altruism, Cheating, and Anticheater Adaptations in Cellular Slime Molds. American Naturalist, 2002, 160 (1), pp.31-43. 10.1086/340613 . hal-02683365

\section{HAL Id: hal-02683365 https://hal.inrae.fr/hal-02683365}

Submitted on 1 Jun 2020

HAL is a multi-disciplinary open access archive for the deposit and dissemination of scientific research documents, whether they are published or not. The documents may come from teaching and research institutions in France or abroad, or from public or private research centers.
L'archive ouverte pluridisciplinaire $\mathbf{H A L}$, est destinée au dépôt et à la diffusion de documents scientifiques de niveau recherche, publiés ou non, émanant des établissements d'enseignement et de recherche français ou étrangers, des laboratoires publics ou privés. 


\title{
Altruism, Cheating, and Anticheater Adaptations in Cellular Slime Molds
}

\author{
Richard Ellis Hudson, ${ }^{\star}$ Juliann Eve Aukema, ${ }^{\dagger}$ Claude Rispe, ${ }^{\ddagger}$ and Denis Roze ${ }^{\S}$
}

Department of Ecology and Evolutionary Biology, University of Arizona, Tucson, Arizona 85721

Submitted September 21, 2000; Accepted December 13, 2001

\begin{abstract}
Cellular slime molds (CSMs) possess a remarkable life cycle that encompasses an extreme act of altruism. CSM cells live as individual amoebae until starved, then aggregate and ultimately transform themselves into a multicellular fruiting body. This fruiting body consists of stalk cells (altruists that eventually die) and spores (the beneficiaries of this sacrifice). Altruistic systems such as this are vulnerable to cheaters, which are individuals unrelated to the altruists that obtain the benefits provided by them without reciprocating. Here, we investigate two forces that can maintain CSM altruism despite cheating: kin selection and anticheater adaptations. First, we present new kinship-based models based on CSM developmental biology to evaluate the efficacy of kin selection. These models show that stalk-making genotypes can still be maintained when aggregations are initiated by multiple "founder" spores, provided that spores of stalkless fruiting bodies have low rates of dispersal and dispersal success is a concave function of stalk height. Second, we review proposals that several features of CSM development, such as the chemical suppression of the redifferentiation of prestalk cells into prespores, act as anticheater adaptations.
\end{abstract}

Keywords: altruism, cellular slime molds, cheater, Dictyostelium, kin selection, multicellularity.

* Corresponding author. Present address: Department of Biology, Indiana University, Bloomington, Indiana 47405; e-mail: rhudson@pobox.com.

+ Present address: Pacific Northwest Research Station, U.S. Department of Agriculture Forest Service, Olympia, Washington 98512; e-mail: jaukema@ alumni.brown.edu.

₹ Present address: Unité Mixte de Recherche Biologie des Organismes et des Populations Appliquée à la Protection des Plantes, Institut National de la Recherche Agronomique, Domaine de la Motte, BP35327, 35653 Le Rheu, France; e-mail: rispe@rennes.inra.fr.

$\checkmark$ Present address: Centre d'Etudes sur le Polymorphisme des Microorganismes Equipe: Evolution Theorique et Experimentale, Institut de Recherche sur le Developpement, 911 avenue Agropolis, BP5045, 34032 Montpellier, France; e-mail: roze@mpl.ird.fr.

Am. Nat. 2002. Vol. 160, pp. 31-43. (c) 2002 by The University of Chicago. 0003-0147/2002/16001-0003\$15.00. All rights reserved.
In nature, altruistic individuals that sacrifice for the benefit of other members of their group are common. Sterile individuals within eusocial insect colonies raise the progeny of their queen, somatic cells within multicellular organisms insure the propagation of the germ line, mitochondria and chloroplasts within eukaryotic cells contribute to the success of the nuclear genome, and DNA polymerases replicate genes of other proteins as well as their own (Szathmáry and Maynard Smith 1995). When cellular slime mold (CSM) amoebae starve, they join together and eventually transform themselves into a fruiting body (fig. 1). Some amoebae become spores, and others become stalk cells; the former disperse, and the latter die while constructing the stalk (Whittingham and Raper 1960). Thus, CSM stalk cells are altruists that, in order to aid spore dispersal, make the greatest sacrifice possible.

Altruists can theoretically be eliminated by cheaters, individuals that obtain the benefits provided by the altruists without reciprocating (Frank 1994; Michod 1997). The establishment and persistence of cheater genotypes require that chimeric groups (groups containing both altruists and cheaters) form with sufficient frequency. Because chimeric CSM groups can form by the unique process of coaggregation of unrelated amoebae, these organisms should be unusually susceptible to cheating. In fact, several cheating CSM strains (strains that have an unusually high propensity to form spores) have been identified (Filosa 1962; Buss 1982; Hilson et al. 1994; Ennis et al. 2000; Strassmann et al. 2000; reviewed in Pál and Papp 2000). To assay for cheaters, spores of two strains are first sown together into one petri dish. A few days later, spores are randomly harvested from the resulting fruiting bodies, and then their genotypes are determined. In early experiments of this type, the ratio of Dictyostelium mucoroides strain DM4-TYPE to strain DM-4-VAR increased from $1: 1$ among the spores sown to $5: 1$ among those harvested, indicating that the former was less likely to form stalk cells (Filosa 1962). In later studies, where harvested spores were used to start subsequent "generations" of fruiting bodies, putative cheaters were greatly enriched 


\section{Life Cycle Stage

Spore
Germinati
spore
Vegetative
amoebae
Aggregat-
ing
ameobae

Simulated Life Cycle:

Stages in the life of one multicellular individual

Aggregation territory contains n spores

Aggregation territory contains n amoebae

Aggregation territory

contains $\mathrm{N}=\mathrm{n} \cdot \mathrm{k}$ amoebae

$\downarrow$ Aggregation

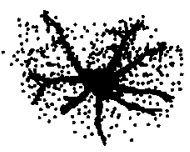

Aggregation

contains $\mathrm{N}=n \cdot k$ amoebae

To spore stage

Some amoebae become prestalk cells (in black, other future support cells also show in black); others become pre-spores (in white)

Pre-stalk cells become stalk cells (in black, other support cells also show in black); pre-spores become spores (in white)

Spores disperse, other cells die

Figure 1: Asexual life cycle and steps of simulated life cycle

in the final harvest (Buss 1982; Ennis et al. 2000). Control experiments have shown that the increase in frequency of putative cheaters was not due to a higher amoeba-stage replication rate (Buss 1982). Although most of these studies employed laboratory mutants, investigations of isolates from soils show that cheating CSM strains occur naturally (Buss 1982; Strassmann et al. 2000). Cheating has also been demonstrated for $M y x$ ococcus xanthus, a eubacterium with a similar life cycle (Velicer et al. 2000).

Cellular slime mold cheater amoebae come in two types. Fixed-allocation cheaters allocate an unusually low proportion of their cells to the stalk in both chimeric and allcheater groups, whereas variable-allocation cheaters allocate a low proportion of their cells to the stalk only when they are in chimeric aggregations (Strassmann et al. 2000). Our models focus on fixed-allocation cheating because variable-allocation cheating should not influence clonal stalk sizes; in theoretical competitions between variableallocation strains, the victor is always a strain that sets its clonal stalk size at the same level selected for in the absence of cheating (Matsuda and Harada 1990).

One might mistakenly conclude that fixed-allocation cheating is unimportant for two reasons: few naturally occurring cheaters are of the fixed-allocation type (Strassmann et al. 2000), and fixed-allocation cheaters should always be eliminated by certain variable-allocation competitors (those that form stalk cells in chimeras with the same propensity as the former but that produce optimalsized stalks in clonal groups). However, although fixed- 
Table 1: Variables and parameters used in the text

\begin{tabular}{ll}
\hline Notation & \\
\hline All models: & Stalk cell to total cell ratio \\
$\alpha, a$ & Number of founders \\
$n$ & Number of descendant amoebas per founder \\
$k$ & Number of cells in an aggregation/slug/fruiting body \\
$N$ & Fitness \\
$W$ & Fecundity \\
$F$ & Dispersibility \\
$D$ & Cost of stalklessness \\
$c$ & Exponent that controls shape of dispersibility function \\
$x$ & Stalk to total cell ratio of a given fruiting body, g \\
$\alpha_{\mathrm{g}}$ & \\
Constant-proportion model: & Constant stalk to spore ratio specified by genotype $j$ \\
$\alpha_{j}$ & Stalk to total cell ratio that maximizes fitness \\
$\alpha_{\mathrm{m}}$ & Evolutionarily stable strategy value of $\alpha$ \\
$\alpha^{*}$ & Kinship coefficient \\
$r$ & \\
Constant-size model: & Stalk cell proportion in all-altruist fruiting body specified by genotype $j$ \\
$a_{j}$ & Stalk cell number in all-altruist fruiting body specified by genotype $j$ \\
$A_{j}$ & Number of altruistic amoebas in an aggregation \\
$N_{\mathrm{a}}$ &
\end{tabular}

allocation cheaters are relatively less common, they are not rare; they comprised one out of 13 soil isolates in one study (Buss 1982) and up to four out of 12 cheaters identified in another (Strassmann et al. 2000). Furthermore, scarcity does not necessarily denote unimportance; the relative dearth of fixed-allocation cheaters may reflect the greater efficacy of kin selection against them than against cheaters of the other type. Regarding the second reason, these competitively superior variable-allocation strains probably do not arise as often as their fixed-allocation counterparts because they have a more complex phenotype; these strains must adjust their allocation in chimeras as a function of the allocation patterns of the other strain(s) in the group (Matsuda and Harada 1990). Furthermore, the ostensibly invincible variable-allocation strains postulated by Matsuda and Harada (1990), which only cheat against strains they recognize as foreign, are still theoretically vulnerable to certain fixed-allocation cheaters (mutants arising from them that they cannot distinguish as alien).

In this article, we describe two kinship-based models of competitions between fixed-allocation cheaters and altruists. Previous kinship-based models of CSMs lack features necessary to evaluate the efficacy of kin selection because they make unrealistic assumptions: altruists in chimeras never become spores (Maynard Smith 1964), fruiting bodies of different sizes have the same dispersal success (Armstrong 1984), and stalkless fruiting bodies have zero fitness (Matapurkar and Watve 1997). Therefore, we have formulated models that relax these assumptions. We then use these models to analyze whether kin selection acting alone might be sufficient to maintain altruism in CSMs, a proposal that has been criticized (Atzmony et al. 1997).

Kin selection prevents the elimination of altruistic genotypes by strengthening between-group selection relative to within-group selection (Sober and Wilson 1998; for applications to specific systems, see Frank 1994; Rispe and Moran 2000). Certain group traits, "anticheater" adaptations, also contribute to the maintenance of altruism via the same mechanism. We close by describing and evaluating several proposals for such adaptations in CSMs.

\section{Kin Selection in Cellular Slime Molds: Two New Models}

Because aggregations descended from two or more CSM spores are probably not rare (Wilson and Sober 1989), we have used our models to explore the level of stalk formation that can be maintained when there are multiple founders. Our models simulate competitions between genotypes that code for different levels of stalk formation in a life cycle that consists of four stages: random association of germinating "founder" spores, growth and aggregation, differentiation, and dispersal (fig. 1; see table 1 for a list of the models' parameters). First, founder spores are randomly distributed into groups of size $n$. In the second stage, each founder gives rise to $k$ descendant amoebae, which then aggregate with the other amoebae of their founder group to produce an aggregation of $N=n k$ cells. (We do not model the movement of foraging amoebae 
because this process should be less important than spore dispersal in generating the spatial distribution of cells.) During the third stage, cells differentiate into stalk cells and spores in accordance with their genotypes; each cell of genotype $j$ has an $\alpha_{j}$ (model 1 ) or $a_{j}$ (model 2) probability of becoming a stalk cell. Finally, spores disperse, with their success depending on the height of their stalk, which is a function of the values of $\alpha_{j}$ (or $a_{j}$ ) of the genotypes that make up each fruiting body.

The fitness of a genotype in a given fruiting body is a function of both the number of its spores and the size of the stalk:

$$
W\left(\alpha_{j}\right)=F\left(\alpha_{j}\right) D\left(\alpha_{\mathrm{g}}\right)
$$

The variables $W\left(\alpha_{j}\right)$ and $F\left(\alpha_{j}\right)$ represent, respectively, the fitness and fecundity (number of spores) of genotype $j$ per founder cell of that genotype, and $D\left(\alpha_{\mathrm{g}}\right)$ stands for the dispersibility of a fruiting body, $\mathrm{g}$, where the proportion of cells that are stalk cells equals $\alpha_{\mathrm{g}}$; the value of $D\left(\alpha_{\mathrm{g}}\right)$ is an increasing function of $\alpha_{\mathrm{g}}$, and $F\left(\alpha_{j}\right)=k\left(1-\alpha_{j}\right)$. Given $i$ different genotypes, $\alpha_{\mathrm{g}}$ is the weighted average of the $i$ values of $\alpha_{j}$. Having $D$ increase with $\alpha_{\mathrm{g}}$ means that having a larger stalk bestows a greater benefit, such as enhanced dispersal (Bonner 1982) or enhanced displacement of spores away from harmful agents in the soil (Gadagkar and Bonner 1994). A $D$ that increases with $\alpha_{\mathrm{g}}$ is also appropriate if prestalk cells provide benefits that increase with their frequency, such as an improved ability to locate and move to better dispersal sites (Inouye and Takeuchi 1980; Miura and Siegert 2000). Although dispersibility increases with $\alpha$, having too many stalk cells can be detrimental because a genotype that increases its $D$ by increasing its $\alpha_{j}$ also decreases its fecundity.

Our formula for $D$ contains two parameters: $c$, the cost of stalklessness, and $x$, which determines the shape of $D$ :

$$
D\left(\alpha_{\mathrm{g}}, c, x\right)=1-c+c \alpha_{\mathrm{g}}^{x}
$$

The parameter $c$ permits the assignment of a nonzero dispersibility to stalkless fruiting bodies. Spores of such fruiting bodies may occasionally have some "dispersal" success; they may be dispersed at a rate lower than that of spores borne on stalks, or they may germinate without being dispersed. Thus, we have set $D\left(\alpha_{\mathrm{g}}=0\right)$ equal to $1-c$; $c$ then represents the cost of having no stalk.

Values of $x$ greater than, equal to, and less than one generate convex, linear, and concave $D$ versus $\alpha$ curves, respectively. (Figure 2 shows the effects of $x$ on fitness.) The shape of the $D$ versus $\alpha$ curve describes where the greatest gains in the dispersibility component of fitness are made; if, for example, dispersal success increases at a
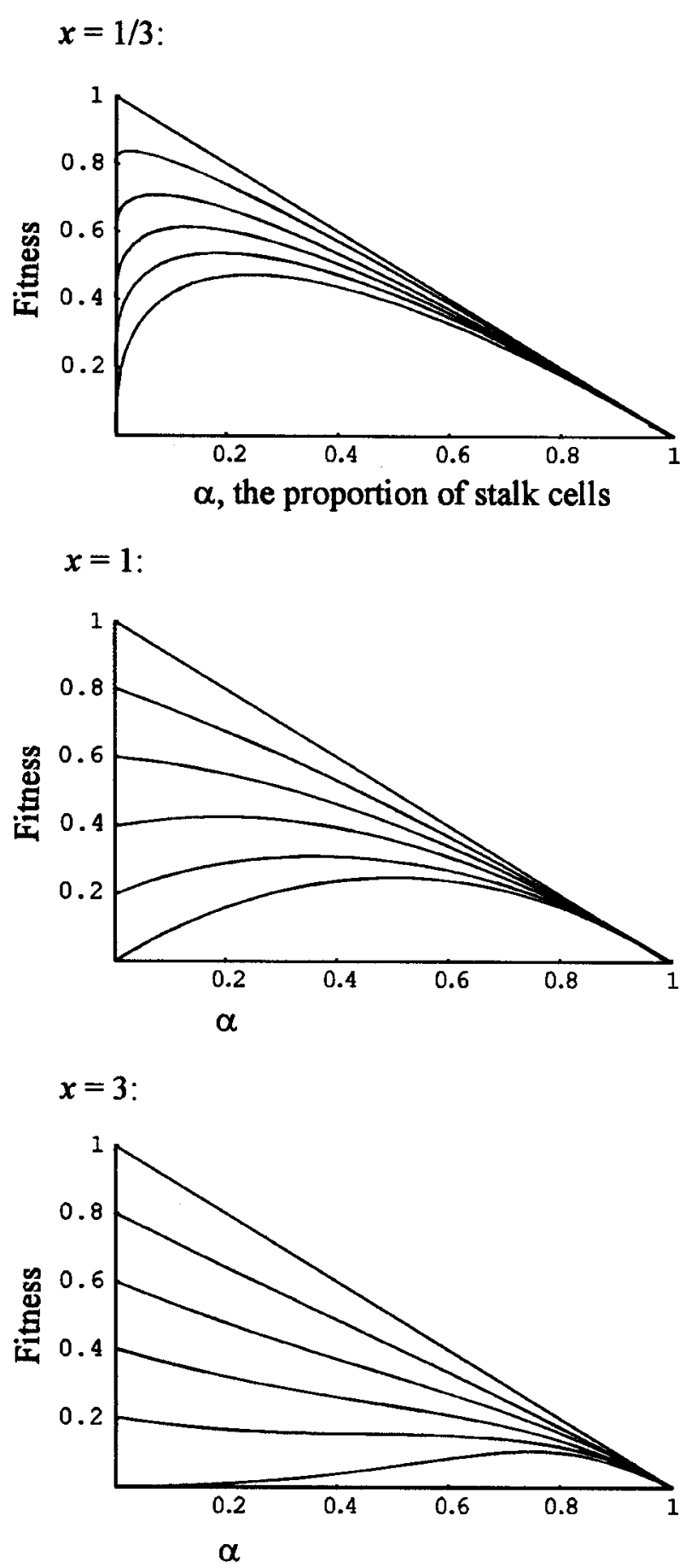

Figure 2: Fitness as a function of $\alpha, c$, and $x$. Within each graph, the curves (from top to bottom) are for $c=0,0.2,0.4,0.6,0.8$, and 1 . This formulation applies to both models. 
higher rate per unit length for taller stalks, then a convex $D$ is most appropriate. Small values of $x$ are most suitable if there is a maximum dispersibility asymptotically approached by taller and taller fruiting bodies.

\section{The Constant-Proportion Model}

We have formulated two models that represent different ways that stalk-making genotypes allocate their cells in chimeric groups. In our first model, each genotype codes for a constant proportion of stalk cells, which is expected when the propensity to become a prestalk cell depends on a cell-autonomous mechanism. In both pure fruiting bodies and chimeras, the fraction of all cells of genotype $j$ that are stalk cells always equals $\alpha_{j}$ (i.e., the presence of cells with other genotypes has no effect on genotype $j$ 's cell allocation).

Within each chimeric group, the genotype with the lower value of $\alpha_{j}$ is cheating on the other genotype, and its frequency increases within groups. Consider two genotypes where $\alpha_{1}<\alpha_{2}$. Suppose that the ratio of genotype 1 amoebae to genotype 2 amoebae is initially $1: 1$; the ratio of their spores in the resulting fruiting body will be $\left(1-\alpha_{1}\right):\left(1-\alpha_{2}\right)>1$.

To analyze the constant-proportion model, we determined the evolutionarily stable strategy (ESS; Maynard Smith and Price 1973), which is the strategy that resists displacement by all other strategies. The ESS is the value of $\alpha^{*}$ that satisfies the following equation:

$$
\left.\frac{\partial W_{\alpha, \alpha^{*}}}{\partial \alpha}\right|_{\alpha=\alpha^{*}}=0
$$

where $W_{\alpha, \alpha^{*}}$ represents the fitness of a founder of genotype $\alpha$ in a group where all the other founders are of genotype $\alpha^{*}$.

No Chimeras, Linear Dispersability $(n=1, x=1)$. In these conditions, the genotype that maximizes fruiting body fitness, $\mathrm{m}$, drives all others to extinction:

$$
\alpha^{*}=\alpha_{\mathrm{m}}= \begin{cases}0 & \text { for } c \leq \frac{1}{2} \\ \frac{2 c-1}{2 c} & \text { for } c>\frac{1}{2}\end{cases}
$$

Equation (4) shows that when $c$, the cost of stalklessness, is low, the stalkless form has the maximum fitness. As the cost of stalklessness increases above one-half, the stalk size that maximizes fitness increases.

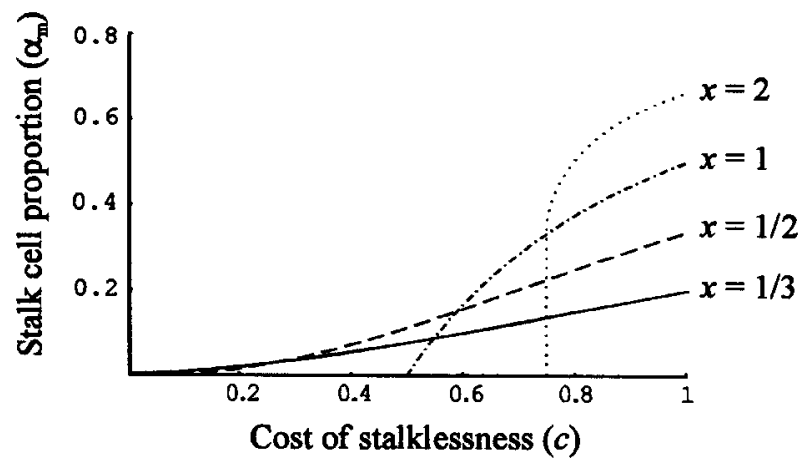

Figure 3: The value of $\alpha$ that maximizes fitness, $\alpha_{\mathrm{m}}$, as a function of $c$ and $x$. The value of $D$ is concave for $x<1$ and convex for $x>1$. The value of $\alpha_{\mathrm{m}}$ is 0 for $x=1, c<0.5$ and for $x=2, c<0.75$.

No Chimeras, Nonlinear Dispersability $(n=1, x \neq 1)$. When $x$ can vary, the value of $\alpha_{\mathrm{m}}$ that maximizes fitness is the solution of the following equation (see also fig. 3; when the solution is negative, $\alpha_{\mathrm{m}}=0$ ):

$$
\alpha_{\mathrm{m}}^{x}-\frac{x}{x+1} \alpha_{\mathrm{m}}^{x-1}+\frac{1-c}{c(x+1)}=0, \text { for } 0<c \leq 1
$$

Varying $x$ affects the outcomes as follows: Stalk-making genotypes have the highest fitness only when $x$ is below a threshold value because only then is the dispersibility of short-stalked fruiting bodies significantly higher than that of stalkless fruiting bodies. This means that concave $D$ functions generally favor altruists and convex $D$ functions generally favor stalkless genotypes. But this does not mean that more extreme concavity always favors higher levels of altruism; in many cases, increasing concavity (by decreasing $x$ ) actually decreases $\alpha_{\mathrm{m}}$ (fig. 3). In such cases, decreasing $x$ decreases the dispersal advantage of intermediatesized stalks relative to small ones and thus selects for smaller stalks.

The parameters $c$ and $x$ interact as follows: As $c$ increases, the threshold value of $x$ increases, making it less likely that the stalkless genotype has the maximum fitness. As $c$ increases for a given $x$, the stalk size that maximizes fitness increases; in other words, larger values of $c$ favor greater degrees of altruism.

Chimeras Form, Linear Dispersability $(n>1, x=1)$. To solve equation (3) for the ESS, we have set $W_{\alpha, \alpha^{*}}$, the fitness of a genotype- $\alpha$ founder in a group where all other founders are of genotype $\alpha^{*}$, equal to the genotype's fecundity, $k(1-\alpha)$, multiplied by the group's dispersibility. When 
$x=1$ and $c=1$, the group's dispersibility equals its stalk cell proportion, $\alpha_{\mathrm{g}}=(1 / n) \alpha+[(n-1) / n] \alpha^{*}$, giving

$$
W_{\alpha, \alpha^{*}}=k(1-\alpha)\left(\frac{1}{n} \alpha+\frac{n-1}{n} \alpha^{*}\right)
$$

Solving for the ESS gives

$$
\alpha^{*}=\frac{1}{1+n} .
$$

The ESS can also be expressed in terms of the kinship coefficient, $r$ (Hamilton 1964, 1970; Frank 1994), which equals $d \alpha_{\mathrm{g}} / d \alpha$. To do so, we take the derivative of equation (6):

$$
\begin{aligned}
\left.\frac{\partial W_{\alpha, \alpha^{*}}}{\partial \alpha}\right|_{\alpha=\alpha^{*}} & =k\left[-\alpha_{\mathrm{g}}+(1-\alpha) \frac{d}{d \alpha} \alpha_{\mathrm{g}}\right] \\
& =k\left[-\alpha^{*}+\left(1-\alpha^{*}\right) r\right] .
\end{aligned}
$$

Setting $\partial W / \partial \alpha=0$ and solving for $\alpha^{*}$ in terms of $r$ gives

$$
\alpha^{*}=\frac{r}{1+r} \text {. }
$$

When each aggregation is founded by only one spore $(n=1)$, the amoebae in one group are all related, kinship is at its maximum $(r=1)$, and the ESS is the same as $\alpha_{\mathrm{m}}$, the value of $\alpha$ that maximizes group fitness. As $n$ increases and kinship decreases, $\alpha^{*}$ decreases, although $\alpha_{\mathrm{m}}$ remains unchanged. The difference between $\alpha^{*}$ and $\alpha_{\mathrm{m}}$ can be thought of as the amount of potential fitness not achieved; this difference grows larger as $n$ increases.

The parameter $k$ is the number of amoebae descended from each founding spore. Because it does not differ among genotypes, the value of $k$ does not affect the value of the ESS nor does it affect the outcomes of the second model.

When $x=1$ but $c \neq 1$, the fitness of strategy $\alpha$ (when rare) becomes

$$
W_{\alpha, \alpha^{*}}=k(1-\alpha)\left[1-c+c\left(\frac{1}{n} \alpha+\frac{n-1}{n} \alpha^{*}\right)\right] .
$$

The ESS for this fitness function is as follows (see also fig. 4):

\section{(A)}

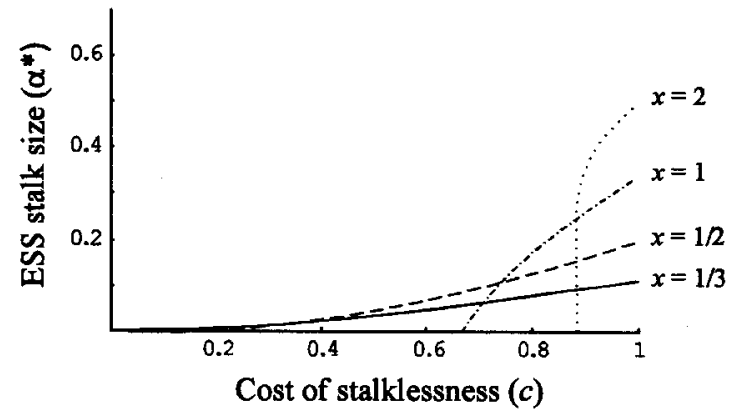

(B)

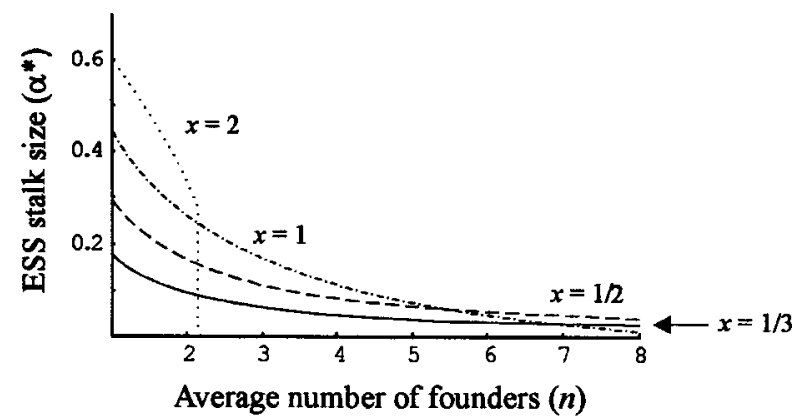

Figure 4: Evolutionarily stable strategy stalk size as a function of $(A) c$ and $x$ (when $n=2$ ) and $(B) n$ and $c$ (when $c=0.9$ ).

$$
\alpha^{*}= \begin{cases}0 & \text { for } c \leq \frac{n}{1+n} \\ \frac{1-(1-c)(1+n)}{(1+n)-(1-c)(1+n)} & \text { for } c>\frac{n}{1+n}\end{cases}
$$

Equation (11) shows that when the cost of stalklessness is low or when the number of founders is high, the ESS is to make no stalk $\left(\alpha^{*}=0\right)$. When $c$ exceeds the $n /(1+n)$ threshold, the ESS increases as $c$ increases but decreases as $n$ increases.

Chimeras Form, Nonlinear Dispersability Functions ( $n>1$, $x \neq 1$ ). When $x$ can vary, the fitness of strategy $\alpha^{*}$ (when rare) becomes

$$
W_{\alpha, \alpha^{*}}=k(1-\alpha)\left[1-c+c\left(\frac{1}{n} \alpha+\frac{n-1}{n} \alpha^{*}\right)^{x}\right] .
$$

The ESS is the solution of the following equation:

$$
\alpha^{x}-\frac{x}{x+n} \alpha^{x-1}+\frac{(1-c) n}{c(x+n)}=0 .
$$


Table 2: Number of different cell types in chimeric fruiting bodies of constant-size model

\begin{tabular}{lcccc}
\hline Total altruist cells & Total stalk cells & Total spores & Cheater-genotype spores $^{\mathrm{a}}$ & Altruist-genotype spores $^{-}$ \\
\hline$N_{\mathrm{a}}\left(<A_{j}\right)$ & $N_{\mathrm{a}}$ & $N-N_{\mathrm{a}}$ & $N-N_{\mathrm{a}}$ & 0 \\
$N_{\mathrm{a}}\left(>A_{j}\right)$ & $A_{j}$ & $N-A_{j}$ & $N-N_{\mathrm{a}}$ & $N_{\mathrm{a}}-A_{j}$ \\
\hline
\end{tabular}

All cheaters become spores.

Varying $x$ has the following effects: when $x$ is low, $\alpha^{*}$ is always $>0$; when $x$ is high, $\alpha^{*}=0$ for much of the parameter space (fig. 4). As before, threshold values of $x$ exist, above which $\alpha^{*}=0$; these threshold values are lower than those when $n=1$ but behave similarly otherwise.

\section{The Constant-Size Model}

It is possible that in chimeras, altruists, being unable to distinguish between themselves and others, increase their probability of becoming stalk cells to compensate for the deficit of cheater-genotype stalk cells. Allocating cells in this way is expected when the propensity of an altruist to become a prestalk cell depends on certain diffusible factors as described in "Discussion." Our second model simulates competitions between altruists employing such an allocation mechanism and a stalkless cheater genotype. In this model, each altruistic genotype is characterized by $a_{j}$ and $A_{j}$, the proportion and number, respectively, of its cells that become stalk cells in all-altruist fruiting bodies:

$$
a_{j}=\frac{\text { stalk cells in pure fruiting body }}{\text { total cells in pure fruiting body }}=\frac{A_{j}}{N} .
$$

In chimeric groups, if the number of altruistic cells $\left(N_{\mathrm{a}}\right)$ is insufficient to form a stalk of $A_{j}$ cells, then all altruists become stalk cells, and if $N_{\mathrm{a}}$ exceeds $A_{j}$, then $A_{j}$ altruists become stalk cells and the rest become spores (table 2).

The constant-size model cannot be analyzed using the ESS approach because in this model, $W_{\alpha, \alpha^{*}}$ is not defined for $\alpha^{*} \neq 0$. Instead, we analyzed pairwise competitions when either cheaters or altruists were rare, with the following possible outcomes: first, cheaters could prevail in both conditions, thus going to fixation; second, altruists could prevail in both conditions; third, both genotypes could increase in frequency when rare, leading to a stable polymorphic equilibrium; and fourth, both genotypes could increase in frequency when common, indicating an unstable polymorphic equilibrium.

The frequency of an altruistic genotype increases when rare only if the fitness of a single altruistic founder in a group of cheaters exceeds the fitness of a single cheating founder in a group of cheaters. To derive the fitness formula for the former, recall that no altruist-genotype amoebae become spores unless $N_{\mathrm{a}}>A_{j}$, then $N_{\mathrm{a}}-A_{j}$ altruist- genotype amoebae become spores. When there is only one altruist-genotype founder, $N_{\mathrm{a}}=k$ and $A_{j}=a_{j} N=a_{j} n k$, which gives the following:

$$
F(\text { lone altruist })=\left\{\begin{array}{ll}
0 & \text { for } k \leq a_{j} n k \\
k-a_{j} n k & \text { for } k>a_{j} n k
\end{array} .\right.
$$

The fitness of the altruistic founder, when $a_{j} n<1$ is then

$$
W=F D=\left(k-k n a_{j}\right)\left(1-c+c a_{j}^{x}\right) .
$$

In the all-cheaters group, the fecundity of one cheatergenotype founder is $k$, and the group's dispersibility is that of a stalkless fruiting body, $1-c$. Thus, the fitness of one cheater-genotype founder is

$$
W=k(1-c)
$$

The altruistic genotype spreads when rare only when the quantity given by equation (16) is greater than the quantity given by equation (17). This inequality simplifies to

$$
\left(\frac{1}{a_{j}^{x}}\right)\left(\frac{a_{j} n}{1-a_{j} n}\right)<\frac{c}{1-c} .
$$

Analogous reasoning shows that the fitness of a lone cheater-genotype founder in a group of altruists always exceeds the fitness of an altruist-genotype founder in an all-altruists group, except for the implausible case where $a_{j}$ is high $(>n /[n-1])$ and the dispersibility function is extremely convex:

$$
\left(D\left(a_{j}\right)>\frac{D(n /(n-1))}{1-a_{j}}\right) .
$$

Therefore, cheaters almost always exist at equilibrium; furthermore, they drive altruists to extinction whenever inequality (18) is false.

Inequality (18) and figure 5 show that for altruists to exist, four conditions must be met. First, $n$ must be relatively low; smaller values of $n$ favor altruists because then fewer chimeric groups form. Second, $c$ must exceed a certain critical value that can be determined by rearranging inequality (18). Third, $x$ must not be too high. As with the other model, the stalkless genotype wins when $x$ ex- 


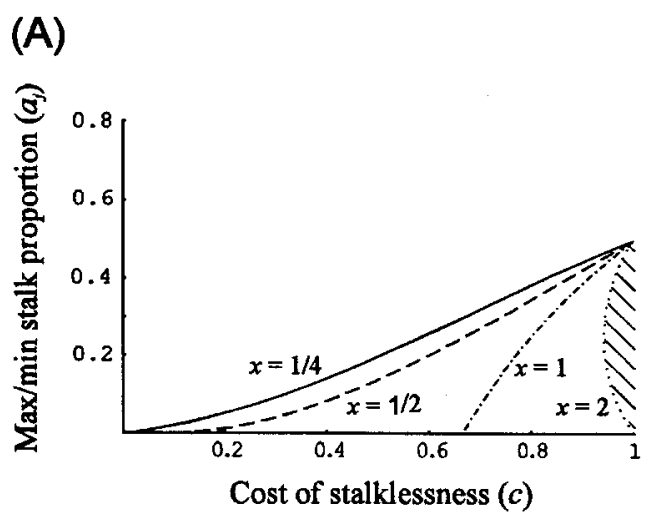

(B)

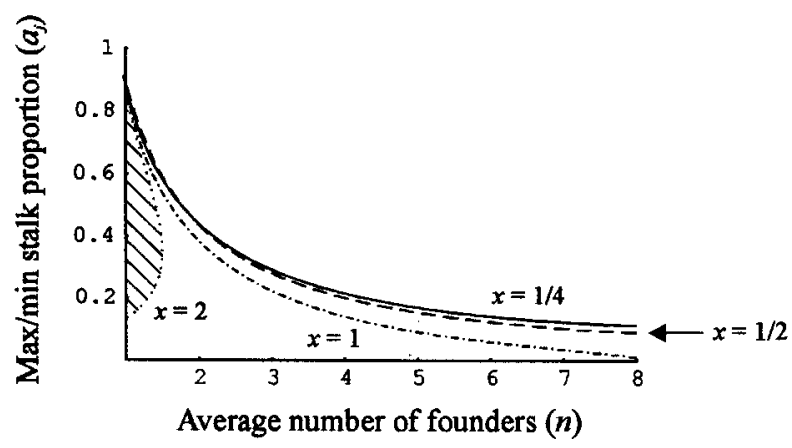

Figure 5: Conditions for the stable existence of altruists in the constantsize model as a function of $(A) c, a_{j}$, and $x(n=2)$ and $(B) n, a_{j}$, and $x(c=0.9)$. For $x=1 / 4,1 / 2$, and 1 , altruists stably coexist with cheaters in the parameter space below the curves (in both graphs), and only cheaters stably persist above these lines. For $x=2$, altruists and cheaters coexist in the striped areas, and only cheaters persist outside these areas.

ceeds a certain threshold, unless $n$ is low and $c$ is very high. Fourth, $a_{j}$ must not be too high (or, in some cases, too low). The variable $a_{j}$ can be thought of as the cost of altruism; it is the minimum proportion of altruists that become stalk cells and therefore die. As with $\alpha^{*}$, the departure of the maximum value of $a_{j}$ from $\alpha_{\mathrm{m}}$ represents the amount of potential fitness not achieved, and this difference increases as $n$ increases and as $c$ decreases. The value of $a_{j}$ never exceeds $1 / n$ and has a minimum value when $x$ is high; in these circumstances, the advantage of having a very short stalk is too small to be worth it.

\section{Sample Calculations}

Our models can be used to determine what values of $c$, $x$, and $n$ are required for kin selection to select for the observed proportion of prestalk cells in clonal groups. In normal Dictyostelium discoideum fruiting bodies, approximately $14 \%$ of the cells are stalk cells and other nonspore cells (Nanjundiah and Bhogle 1995). Since prestalk cells are lost during slug migration and then replaced, $14 \%$ represents a minimum value for the initial prestalk cell fraction. Therefore, we will assume that the initial prestalk fraction $\left(\alpha_{\mathrm{m}}, \alpha^{*}\right.$, or $a_{j}$, depending on the model used) is approximately $20 \%$.

For kin selection alone to produce a value for $\alpha_{\mathrm{m}} / \alpha^{*} / a_{j}$ of $0.2, c$ must be relatively high, $x$ must be in certain ranges, and $n$ must be relatively low. For the case when no chimeras form, $\alpha_{\mathrm{m}}$ never equals 0.2 when $x<0.25$ or $x>$ 1.5. In the former case, $\alpha_{\mathrm{m}}$ is always smaller; in the latter case, $\alpha_{\mathrm{m}}$ is either 0 or $>0.2$. For the stalk cell fraction $\alpha_{\mathrm{m}}$ to reach 0.2 , it is necessary, but not sufficient, that $c \geq$ 0.6 . In the constant-proportion model, $\alpha^{*}$ can reach 0.2 when there are more than one founders, if $c$ is high and if $x$ is intermediate. For example, when $x=1 / 2$ and $c=0.9$, an ESS of 0.2 is achieved when $n=1.6$; lower values of $n$ produce larger ESSs. Similarly, in the constantsize model, altruists with $a_{j}=0.2$ can exist when $n>1$, provided that $c$ is large and $x$ is not too large (table 3 ).

The outcomes for the constant-proportion and constantsize models differ when $x$ is low; in the former, altruists with a stalk cell proportion of 0.2 are always eliminated by shorter-stalked altruists; in the latter, such altruists can still persist. This difference arises because genotypes that make stalks of different sizes can compete against one another in the constant-proportion model but cannot in the constant-size model; in this model, one of the competing strains is always the stalkless strain. Thus, in the constant-size model when $x$ is low, altruists with $a_{j}=$ 0.2 are not eliminated by strains forming shorter stalks because they do not face them in direct competition.

\section{Discussion}

Our models show that competitions involving fixedallocation cheaters select for suboptimal stalks, in contrast to theoretical competitions involving variable-allocation strains, where clonal stalks of suboptimal size are not selected for (Matsuda and Harada 1990). The ratio of CSM

Table 3: Maximum values of $n$ that allow altruists with $a_{j}=$ 0.2 to coexist with cheaters at equilibrium

\begin{tabular}{lcc}
\hline$x$ & $c$ & Maximum $n$ \\
\hline .25 & .5 & 2.0 \\
.25 & .9 & 4.3 \\
.5 & .5 & 1.5 \\
.5 & .9 & 4.0 \\
1.0 & .5 & $\mathrm{NA}^{\mathrm{a}}$ \\
1.0 & .9 & 3.2 \\
\hline
\end{tabular}

${ }^{\text {a }}$ No altruists at equilibrium. 
prestalk cells to prespores, $1: 5$, is very low; this suggests that natural selection has acted to reduce CSM stalk size.

Although the effects of $n$ and $c$ on expected stalk size are straightforward-increasing the former and decreasing the latter reduces the stalk size selected for-the effects of the shape of dispersibility curve are complicated. High values of $x$, encoding convex dispersibility functions, generally make it harder to select for a stalk. However, when stalks are selected for and when $x$ is below a threshold value, higher values of $x$ tend to favor larger stalks, as indicated by the increases in $\alpha^{*}$ and in the minimum value for $a_{j}$.

Each of our models can be related to specific mechanisms that control differentiation into stalk cells and spores. The initial differentiation of amoebae is influenced by an amoeba's cell-cycle position; amoebae that at the onset of starvation are in mid- and late-growth-2 (G2) stage normally become prespores, and amoebae in other stages normally become prestalk cells (Zimmerman and Weijer 1993; Gomer and Amman 1996; Araki and Maeda 1998; for a review, see Brown and Firtel 1999). Consider a hypothetical mutant with an extended G2 stage but a normal-length cell cycle, which would produce a shortstalked clonal fruiting body. In chimeras, cells of mutant and nonmutant strains should differentiate with the same propensities as they would have in pure fruiting bodies, as in our constant-proportion model, because the differentiation of each cell is controlled autonomously, by its cell-cycle position and its genotype.

Cheating on another regulatory mechanism should lead to the dynamics of the constant-size model. Prestalk cells have the ability to redifferentiate into prespores, and vice versa (Bonner 1982), apparently under the control of a secreted factor that controls gene expression, the differentiation-inducing factor (DIF-1; Gross 1994). The concentration of this factor most likely is regulated by a feedback mechanism arising from its simultaneous production and degradation, respectively, by prespores and prestalk cells (Brookman et al. 1987; Kay et al. 1993). When the concentration of DIF-1 rises, the prestalk cell population should increase, and the prespore population should decrease, thus increasing DIF-1 degradation capacity and decreasing DIF-1 production capacity (Insall et al. 1992; Loomis 1993). Suppose that a mutant that only forms prespores arises and that its prespores produce DIF-1 at the usual rate. In chimeras of this mutant and a wild-type strain, the number of wild-type cells that become prestalk cells should equal that number in all-wild-type fruiting bodies, as postulated by our constant-size model, because the rates of DIF-1 production and degradation are the same as in the wild-type slug.

Given that fixed-allocation cheating is possible, why do CSMs make any stalks at all? Our analyses show that kin selection is sufficient to maintain the observed level of stalk formation even if there are multiple founders, provided that $n$ is low, $x$ is small, and $c$ is large. The number of founders, $n$, should be low when spores are very widely dispersed, when amoebae descended from different founders avoid coaggregation (Buss 1982), or when germinating spores inhibit the germination of their neighbors, as has been demonstrated for young spores (Russell and Bonner 1960; Cohen and Ceccarini 1967). Determining which values of $x$ are most realistic awaits the identification of the mechanisms of spore dispersal, but concave dispersibility functions are not implausible. The cost of stalklessness, $c$, should be large if spores on short stalks are unlikely to be dispersed and if survival rates at the (potentially exhausted) site of the parent fruiting body are lower than those elsewhere.

\section{Anticheater Adaptations}

As noted above, the evolutionary maintenance of altruism is facilitated in theory by phenomena that diminish the amount of selection within groups relative to that between groups. The putative anticheater adaptations described below do this in one of two ways: by reducing the frequency of chimeric groups or by reducing the fitness advantage of cheating within chimeras.

\section{Reducing the Probability of Forming Chimeras}

CSM strains with somatic-compatibility systems, the first of three chimera-avoidance mechanisms, avoid aggregating with cells of other genotypes (Buss 1982). Doing so should be especially effective against cheating when cheating strains are preferentially avoided, as was the case in Buss's (1982) coculture experiments. Models by Armstrong (1984) and Matapurkar and Watve (1997) show how a second mechanism could work: the advantage of altruism is enhanced when the distance that amoebae travel as they forage is reduced. Third, reducing the size of aggregation territories (the territories within which all amoebae coaggregate to form one fruiting body; Bonner and Dodd 1962) should decrease the probability that descendants from different founders coaggregate. Aggregation territory size is negatively correlated with the concentration of a secreted factor, countin (Brock and Gomer 1999), and thus is under genetic control.

\section{Reducing the Fitness Advantage of Cheating within Chimeric Groups}

Randomizing Which Cells Become Prespores. The three putative anticheater adaptations in this category (using cell quality to determine cell fate, coercing low-energy cells 
into differentiating, and using cell-cycle position as a secondary determinant of cell fate) act against two types of cheaters: those that manipulate the fate-determination mechanisms to produce more prespores and those that cheat by foregoing these mechanisms.

An important determinant of an amoeba's fate is its level of energy reserves; amoebae with more mitochondria or with higher levels of stored carbohydrates are more likely to become prespores (Noce and Takeuchi 1985; Shaulsky and Loomis 1995). Cheating by strains that adhere to this system is suppressed because the determination of cell fate acts at random with respect to genotype for strains that produce cells with comparable energy reserves (Gadagkar and Bonner 1984). Cheaters (and altruists) that do not use reserve levels to determine cell fate should also suffer a penalty, if the advantage of allocating high-energy cells to the spore fraction (increased spore survival) outweighs the structural disadvantage of using low-energy cells for the stalk.

It has been proposed that high-quality cells (those with the greatest energy reserves) secrete DIF-1, a toxin, to coerce low-quality cells into becoming prestalk cells (Atzmony et al. 1997). Secretion occurs upon starvation, when the only alternatives available to amoebae are to aggregate (and differentiate) or to remain solitary and face certain death. The high-quality cells would be able to simultaneously detoxify this compound and differentiate into prespores, but low-quality cells would not and would become prestalk cells by default. Cheaters abstaining from toxin degradation to free up resources for differentiating into prespores would be penalized because they would be more susceptible to the toxin.

Although DIF-1 appears too late to initiate differentiation (Shaulsky and Loomis 1996), as required by this scenario, another molecule, cyclic adenosine monophosphate (cAMP), may fulfill the role proposed for this toxin. Cyclic AMP is the molecule responsible for initiating differentiation (Firtel 1995) and is initially secreted only by the amoebae most likely to become prespores (Huang et al. 1997). In addition, although cAMP is not a toxin, it does coerce low-quality cells to respond to it by penalizing them if they do not; amoebae that disregard this signal must also forego aggregation (Firtel 1995). Thus, cAMP, like the toxin in the scenario above, is initially secreted by high-quality cells, initiates differentiation, induces lowquality cells to become prestalk cells, and penalizes cheaters that ignore it.

As noted previously, a cell's fate is largely determined by its cell-cycle position at the onset of starvation. Among amoebae with similar life cycle durations, this system for allocating cells acts at random with respect to genotype and therefore should suppress cheating (Wilson and Sober 1989). Differences in energy-reserve levels among cells in different cell-cycle positions most likely underlie this effect (Gadagkar and Bonner 1994).

One would expect that a cheating mutant that arrests its development during the late G2 phase (and thus locks in a high level of energy reserves) would insure that it would become a prespore. However, this type of cheating would be penalized, if starvation were not imminent, because wild-type cells would continue to divide while the cheaters remained idle. A cheating mutant that decouples its fate from its cell-cycle stage should suffer the same penalty as one that decouples its fate from its energyreserve level because cell-stage and reserve levels are correlated.

Dividing Asymmetrically to Insure That Some Altruists Become Spores. Sister Dictyostelium discoideum amoebae, upon starvation, differentiate into different types of cells, and amoebae that become prestalk cells almost always have sisters that eventually become spores (Gomer and Firtel 1987). If differentiation of altruist-genotype sisters operates in the same way in chimeric fruiting bodies, then it should decrease the fitness advantage of all types of cheaters by increasing the probability that at least one of each pair of altruist-strain sister amoebae will become a prespore.

In Gomer and Firtel's (1987) study, divisions that produced daughters with different predispositions occurred not only during the aggregation phase but also during the vegetative phase, prior to starvation. This suggests that even this solitary phase of the CSM life cycle encompasses a feature that is an anticheater adaptation.

Policing to Prevent Prestalk Cells from Abandoning Their Fate. Incipient prestalk cells unrelated to most prespores in their group should increase their inclusive fitness by redifferentiating into prespores. In theory, policing for such transitions (i.e., detecting and eliminating them) is advantageous at the group level and can be selected for (Frank 1995; Michod 1996). In CSM mounds and slugs, DIF-1 inhibits the redifferentiation of prestalk cells into prespores (Firtel 1995); therefore, this compound may be considered a policing agent. This interpretation can explain several phenomena: the cells that would require policing (prestalk cells) are the ones most sensitive to DIF1; DIF-1 is not secreted until after differentiation has started, which is when the need for a policing agent first arises; and the cells that would benefit most from the policing agent (prespores) are the ones that produce it.

\section{Conclusions}

Kin-selection and anticheater adaptations may seem like alternative explanations for the maintenance of CSM al- 
truism. Models of policing adaptations models do suggest that effective kin selection may hinder the evolution of anticheater adaptations by rendering them unnecessary (Frank 1995, 1996). However, there is no reason to believe that kin selection and anticheater adaptations are incapable of operating concurrently. In fact, many of the putative anticheater adaptations, such as aggregation incompatibility systems, reduction of foraging distances, decreasing aggregation territory size, and asymmetric cell division, work only within the context of kin selection.

The proposition that kin selection maintains CSM altruism has been criticized on several grounds (Atzmony et al. 1997). First, kin selection acting alone may be too ineffective when groups have multiple founders and relatedness is low. However, our sample calculations show that it can maintain the observed level of stalk formation when the average number of founders exceeds one, provided that the fitness of stalkless fruiting bodies is low and dispersal success is a concave function of stalk height. Second, kin selection, it has been argued, cannot explain the function of the degradation of DIF-1 by prestalk cells; however, this process is necessary to generate the concentration gradient required, even in clonal groups, to regulate the proportion of different cell types. A third criticism, that the operation of kin selection is inconsistent with the alleged scarcity of cheaters, has been invalidated by evidence to the contrary (Strassmann et al. 2000).

CSM altruism, we contend, has been maintained in part by a reduced average stalk size, which enables kin selection to select against even more drastic cheating. Selection for smaller stalks has probably driven the evolution of two related traits: increasing the relative duration of the G2 cell-cycle stage and selecting for strains in which only incipient prespores initiate the release of cAMP.

The adaptive function of several other CSM traits, we have argued, is not to facilitate development within clonal organisms, as might be expected, but instead is to suppress both fixed- and variable-allocation cheating. These traits are the following: the exclusion of unrelated amoebae from aggregations (which increases kinship within groups); the regulation of aggregation territory size (which can increase within-group kinship); the use of energy-reserve levels and cell-cycle position to determine an amoeba's fate (which randomizes which cells become prespores); the integration of aggregation and differentiation such that they are initiated by the same signal, cAMP (which forces low-quality amoebae to differentiate into prestalk cells); the asymmetric division of amoebae (which insures that at least one of every pair of starving sisters becomes a spore); and the secretion of DIF-1 in slugs by prespores (which inhibits the redifferentiation of prestalk cells into prespores). We have also proposed that one other trait that suppresses cheating should exist: the reduction of amoebae foraging distances below optimal levels.

The prominence of the features listed above suggests that the potential for cheating has profoundly shaped cellular slime mold biology. In other phylogenetic lineages, similar anticheater adaptations have played a significant role in the evolution of multicellularity (Buss 1987; Michod 1996). Among single-celled organisms, cooperation is prevalent; examples include biofilms of Pseudomonas or Rhodobacter bacteria, coinfecting Salmonella and Staphylococcus cells, and communities of Rhizobia within and outside of legume root nodules (Crespi 2001). As with CSMs, kinship and anticheater adaptations should play a significant role in maintaining the altruism of these organisms.

\section{Acknowledgments}

We are indebted to J. Waage for his encouragement and assistance to J.E.A. on her earlier paper on this subject and to R. E. Michod and the National Institute of Health (grant GM 55505) for supporting R.E.H. throughout this research. We also thank R. E. Michod and S. Richardson for many helpful comments on the manuscript and the reviewers of a previous version of this manuscript for their suggestions.

\section{Literature Cited}

Araki, T., and Y. Maeda. 1998. Mutual relation between the cell-cycle progression and prespore differentiation in Dictyostelium development. Zoological Science (Tokyo) 15:77-84.

Armstrong, D. P. 1984. Why don't cellular slime molds cheat? Journal of Theoretical Biology 109:271-283.

Atzmony, D., A. Zahavi, and V. Nanjundiah. 1997. Altruistic behaviour in Dictyostelium discoideum explained on the basis of individual selection. Current Science (Bangalore) 72:142-145.

Bonner, J. T. 1982. Evolutionary strategies and developmental constraints in the cellular slime molds. American Naturalist 119:530-552.

Bonner, J. T., and M. R. Dodd. 1962. Aggregation territories in the cellular slime molds. Biological Bulletin (Woods Hole) 122:13-24.

Brock, D. A., and R. H. Gomer. 1999. A cell-counting factor regulating structure size in Dictyostelium. Genes \& Development 13:1960-1969.

Brookman, J. J., K. A. Jermyn, and R. R. Kay. 1987. Nature and distribution of the morphogen DIF in the Dictyostelium slug. Development (Cambridge) 100:119-124.

Brown, J. M., and R. A. Firtel. 1999. Regulation of cellfate determination in Dictyostelium. Developmental Biology 216:426-441. 
Buss, L. W. 1982. Somatic-cell parasitism and the evolution of somatic tissue compatibility. Proceedings of the National Academy of Sciences of the USA 79:5337-5341.

- 1987. The evolution of individuality. Princeton University Press, Princeton, N.J.

Cohen, A., and C. Ceccarini. 1967. Inhibition of spore germination in the cellular slime moulds. Annals of Botany (London) 31:479-487.

Crespi, B. J. 2001. The evolution of social behavior in microorganisms. Trends in Ecology \& Evolution 16: 178-183.

Ennis, H. L., D. N. Dao, S. U. Pukatzki, and R. H. Kessin. 2000. Dictyostelium amoebae lacking an F-box protein form spores rather than stalk in chimeras with wild type. Proceedings of the National Academy of Sciences of the USA 97:3292-3297.

Filosa, M. F. 1962. Heterocytosis in cellular slime molds. American Naturalist 96:79-91.

Firtel, R. A. 1995. Integration of signaling information in controlling cell-fate decisions in Dictyostelium. Genes \& Development 9:1427-1444.

Frank, S. A. 1994. Kin selection and virulence in the evolution of protocells and parasites. Proceedings of the Royal Society of London B, Biological Sciences 258: 153-161.

- 1995. Mutual policing and repression of competition in the evolution of cooperative groups. Nature 377:520-522.

- 1996. Policing and group cohesion when resources vary. Animal Behaviour 53:1163-1169.

Gadagkar, R., and J. T. Bonner. 1994. Social insects and social amoebae. Journal of Biosciences (Bangalore) 19: 219-245.

Gomer, R. H., and R. R. Ammann. 1996. A cell-cycle phase-associated cell-type choice mechanism monitors the cell cycle rather than using an independent timer. Developmental Biology 174:82-91.

Gomer, R. H., and R. A. Firtel. 1987. Cell-autonomous determination of cell-type choice in Dictyostelium development by cell-cycle phase. Science (Washington, D.C.) $237: 758-762$.

Gross, J. D. 1994. Developmental decisions in Dictyostelium discoideum. Microbiological Reviews 58:330-351.

Hamilton, W. D. 1964. The genetical evolution of social behavior. International Journal of Theoretical Biology $7: 1-16$.

- 1970. Selfish and spiteful behaviour in an evolutionary model. Nature 269:1218-1220.

Hilson, J. A., S. A. Kolmes, and L. F. Nellis. 1994. Fruiting body architecture, spore capsule contents, selfishness, and heterocytosis in the cellular slime mold Dictyostelium discoideum. Ethology Ecology \& Evolution 6:529-535.

Huang, H. J., D. Takagawa, G. Weeks, and C. Pears. 1997.
Cells at the center of Dictyostelium aggregates become spores. Developmental Biology 192:564-571.

Inouye, K., and I. Takeuchi. 1980. Motive force of the migrating pseudoplasmodium of the cellular slime mould Dictyostelium discoideum. Journal of Cell Science 41: 53-64.

Insall, R., O. Nayler, and R. R. Kay. 1992. DIF-1 induces its own breakdown in Dictyostelium. EMBO (European Molecular Biology Organization) Journal 11:2849-2854.

Kay, R. R., S. Large, D. Traynor, and O. Nayler. 1993. A localized differentiation-inducing-factor sink in the front of the Dictyostelium slug. Proceedings of the National Academy of Sciences of the USA 90:487-491.

Loomis, W. F. 1993. Lateral inhibition and patternformation in Dictyostelium. Current Topics in Developmental Biology 28:1-46.

Matapurkar, A. K., and M. G. Watve. 1997. Altruist cheater dynamics in Dictyostelium: aggregated distribution gives stable oscillations. American Naturalist 150:790-797.

Matsuda, H., and Y. Harada. 1990. Evolutionarily stable stalk to spore ratio in cellular slime molds and the law of equalization in net incomes. Journal of Theoretical Biology 147:329-344.

Maynard Smith, J. 1964. Group selection and kin selection. Nature 201:1145-1147.

Maynard Smith, J., and G. R. Price. 1973. The logic of animal conflict. Nature 246:15-18.

Michod, R. E. 1996. Cooperation and conflict in the evolution of individuality. II. Conflict mediation. Proceedings of the Royal Society of London B, Biological Sciences 263:813-822.

- 1997. Cooperation and conflict in the evolution of individuality. I. Multilevel selection of the organism. American Naturalist 149:607-645.

Miura, K., and F. Siegert. 2000. Light affects cAMP signaling and cell movement activity in Dictyostelium discoideum. Proceedings of the National Academy of Sciences of the USA 97:2111-2116.

Nanjundiah, V., and A. S. Bhogle. 1995. The precision of regulation in Dictyostelium discoideum: implications for cell-type proportioning in the absence of spatial pattern. Indian Journal of Biochemistry and Biophysics 32:404-416.

Noce, T., and I. Takeuchi. 1985. Prestalk prespore differentiation tendency of Dictyostelium discoideum cells as detected by a stalk-specific monoclonal antibody. Developmental Biology 109:157-164.

Pál, C., and B. Papp. 2000. Selfish cells threaten multicellular life. Trends in Ecology \& Evolution 15:351-352.

Rispe, C., and N. A. Moran. 2000. Accumulation of deleterious mutations in endosymbionts: Muller's ratchet with two levels of selection. American Naturalist 156: 425-441. 
Russell, G. K., and J. T. Bonner. 1960. A note on spore germination in the cellular slime mold Dictyostelium mucoroides. Bulletin of the Torrey Botanical Club 87: 187-191.

Shaulsky, G., and W. F. Loomis. 1995. Mitochondrial-DNA replication but no nuclear-DNA replication during development of Dictyostelium. Proceedings of the National Academy of Sciences of the USA 92:5660-5663.

. 1996. Initial cell type divergence in Dictyostelium is independent of DIF-1. Developmental Biology 174: 214-220.

Sober, E., and D. S. Wilson. 1998. Unto others: the evolution and psychology of unselfish behavior. Harvard University Press, Cambridge, Mass.

Strassmann, J. E., Y. Zhu, and D. Queller. 2000. Altruism and social cheating in the social amoeba Dictyostelium discoideum. Nature 408:965-967.
Szathmáry, E., and J. Maynard Smith. 1995. The major evolutionary transitions. Nature 374:227-232.

Velicer, G. J., L. Kroos, and R. E. Lenski. 2000. Developmental cheating in the social bacterium Myxococcus xanthus. Nature 404:598-601.

Whittingham, W. F., and K. B. Raper. 1960. Non-viability of stalk cells in Dictyostelium. Proceedings of the National Academy of Sciences of the USA 46:642-649.

Wilson, D. S., and E. Sober. 1989. Reviving the superorganism. Journal of Theoretical Biology 136:337-356.

Zimmerman, W., and C. J. Weijer. 1993. Analysis of cellcycle progression during the development of Dictyostelium and its relationship to differentiation. Developmental Biology 160:178-185.

Associate Editor: David E. McCauley 